\title{
Boundary triples for Schrödinger operators with singular interactions on hypersurfaces
}

\author{
J. Behrndt ${ }^{1}$, M. Langer ${ }^{2}$ and V. Lotoreichik ${ }^{3}$ \\ ${ }^{1}$ Institut für Numerische Mathematik, Technische Universität Graz, \\ Steyrergasse 30, 8010 Graz, Austria \\ ${ }^{2}$ Department of Mathematics and Statistics, University of Strathclyde, \\ 26 Richmond Street, Glasgow G1 1XH, United Kingdom \\ ${ }^{3}$ Department of Theoretical Physics, Nuclear Physics Institute CAS, \\ 25068 Řež near Prague, Czech Republic \\ behrndt@tugraz.at,m.langer@strath.ac.uk, lotoreichik@ujf.cas.cz
}

PACS 02.30.Tb, 03.65.Db

DOI 10.17586/2220-8054-2016-7-2-290-302

\begin{abstract}
The self-adjoint Schrödinger operator $A_{\delta, \alpha}$ with a $\delta$-interaction of constant strength $\alpha$ supported on a compact smooth hypersurface $\mathcal{C}$ is viewed as a self-adjoint extension of a natural underlying symmetric operator $S$ in $L^{2}\left(\mathbb{R}^{n}\right)$. The aim of this note is to construct a boundary triple for $S^{*}$ and a self-adjoint parameter $\Theta_{\delta, \alpha}$ in the boundary space $L^{2}(\mathcal{C})$ such that $A_{\delta, \alpha}$ corresponds to the boundary condition induced by $\Theta_{\delta, \alpha}$. As a consequence, the well-developed theory of boundary triples and their Weyl functions can be applied. This leads, in particular, to a Krein-type resolvent formula and a description of the spectrum of $A_{\delta, \alpha}$ in terms of the Weyl function and $\Theta_{\delta, \alpha}$.
\end{abstract}

Keywords: Boundary triple, Weyl function, Schrödinger operator, singular potential, $\delta$-interaction, hypersurface.

Received: 22 January 2016

\section{Introduction}

Boundary triples and their Weyl functions are efficient and frequently used tools in the extension theory of symmetric operators and the spectral analysis of their self-adjoint extensions. Roughly speaking, a boundary triple consists of two boundary mappings that satisfy an abstract second Green's identity and a maximality condition. With the help of a boundary triple, all self-adjoint extensions of a symmetric operator can be parameterized via abstract boundary conditions that involve a self-adjoint parameter in a boundary space. In addition, the spectral properties of these self-adjoint extensions can be described with the help of the Weyl function and the corresponding boundary parameters. We refer the reader to [1-5] and Section 2 for more details on boundary triples and their Weyl functions.

The main objective of this note is to provide and discuss boundary triples and their Weyl functions for self-adjoint Schrödinger operators in $L^{2}\left(\mathbb{R}^{n}\right)$ with $\delta$-interactions of strength $\alpha \in \mathbb{R}$ supported on a compact smooth hypersurface $\mathcal{C}$ that separates $\mathbb{R}^{n}$ into a smooth bounded domain $\Omega_{i}$ and an unbounded smooth exterior domain $\Omega_{e}$. In an informal way, such an operator is often written in the form

$$
A_{\delta, \alpha}=-\Delta-\alpha \delta_{\mathcal{C}}
$$

where $\delta_{\mathcal{C}}$ denotes the $\delta$-distribution supported on $\mathcal{C}$. A precise definition of the self-adjoint operator $A_{\delta, \alpha}$ in terms of boundary or interface conditions is given at the beginning of Section 3 below; see also $[6,7]$ for an equivalent definition via quadratic forms. Schrödinger operators with $\delta$-interactions are frequently used in mathematical physics to model interactions of quantum particles; we refer to the monographs [8] and [9], to the review article [10] and to [6,11-25] for a small selection of related papers on spectral analysis of such operators. 
Let $A_{\text {free }}$ be the usual self-adjoint realization of $-\Delta$ in $L^{2}\left(\mathbb{R}^{n}\right)$ and let $A_{\delta, \alpha}$ be the self-adjoint operator with $\delta$-interaction on $\mathcal{C}$ in (1). We consider the densely defined, closed symmetric operator $S=A_{\text {free }} \cap A_{\delta, \alpha}$ in $L^{2}\left(\mathbb{R}^{n}\right)$ and its adjoint $S^{*}$, and we construct a boundary triple $\left\{L^{2}(\mathcal{C}), \Gamma_{0}, \Gamma_{1}\right\}$ for $S^{*}$ and a self-adjoint parameter $\Theta_{\delta, \alpha}$ in $L^{2}(\mathcal{C})$ such that

$$
A_{\text {free }}=S^{*} \uparrow \operatorname{ker} \Gamma_{0} \quad \text { and } \quad A_{\delta, \alpha}=S^{*} \uparrow \operatorname{ker}\left(\Gamma_{1}-\Theta_{\delta, \alpha} \Gamma_{0}\right) .
$$

Although it is clear from the general theory that such a boundary triple and a self-adjoint parameter $\Theta_{\delta, \alpha}$ exist, its construction is not trivial. Our idea here is based on a coupling of two boundary triples for elliptic PDEs which involve the Dirichlet-to-Neumann map as a regularization (see [26-28]), the restriction of this coupling to a suitable intermediate extension, and certain transforms of boundary triples and corresponding parameters. These efforts and technical considerations are worthwhile for various reasons. In particular, if $\gamma$ and $M$ denote the $\gamma$-field and Weyl function corresponding to the boundary triple $\left\{L^{2}(\mathcal{C}), \Gamma_{0}, \Gamma_{1}\right\}$ (see Section 2 for more details), then it follows immediately from the general theory in $[3,4]$ that the resolvent difference of $A_{\text {free }}$ and $A_{\delta, \alpha}$ admits the representation

$$
\left(A_{\delta, \alpha}-\lambda\right)^{-1}-\left(A_{\text {free }}-\lambda\right)^{-1}=\gamma(\lambda)\left(\Theta_{\delta, \alpha}-M(\lambda)\right)^{-1} \gamma(\bar{\lambda})^{*}
$$

for all $\lambda \in \rho\left(A_{\delta, \alpha}\right)$ and belongs to some operator ideal in $L^{2}\left(\mathbb{R}^{n}\right)$ if and only if the resolvent of $\Theta_{\delta, \alpha}$ belongs to the analogous operator ideal in $L^{2}(\mathcal{C})$; see Theorem 3.5. As a special case, the Schatten-von Neumann properties of the resolvent difference of $A_{\text {free }}$ and $A_{\delta, \alpha}$ carry over to the resolvent of $\Theta_{\delta, \alpha}$, and vice versa. Moreover, the spectral properties of $A_{\delta, \alpha}$ can be described with the help of the perturbation term $\left(\Theta_{\delta, \alpha}-M(\lambda)\right)^{-1}$. We mention that in the context of the more general notion of quasi boundary triples and their Weyl functions from $[29,30]$ a similar approach as in this note and closely related results can be found in [6,31]; we also refer to $[27,28,32-37]$ for other methods in extension theory of elliptic differential operators.

\section{Boundary triples and Weyl functions}

In this preparatory section, we recall the notion of boundary triples, associated $\gamma$-fields and Weyl functions, and discuss some of their properties. For a more detailed exposition, we refer the reader to $[1-5,38]$.

In the following, let $\mathfrak{H}$ be a Hilbert space, let $S$ be a densely defined, closed symmetric operator in $\mathfrak{H}$, and let $S^{*}$ be the adjoint operator.

Definition 2.1. A triple $\left\{\mathcal{G}, \Gamma_{0}, \Gamma_{1}\right\}$ is called a boundary triple for $S^{*}$ if $\mathcal{G}$ is a Hilbert space and $\Gamma_{0}, \Gamma_{1}: \operatorname{dom} S^{*} \rightarrow \mathcal{G}$ are linear mappings that satisfy the abstract second Green's identity

$$
\left(S^{*} f, g\right)_{\mathfrak{H}}-\left(f, S^{*} g\right)_{\mathfrak{H}}=\left(\Gamma_{1} f, \Gamma_{0} g\right)_{\mathcal{G}}-\left(\Gamma_{0} f, \Gamma_{1} g\right)_{\mathcal{G}}
$$

for all $f, g \in \operatorname{dom} S^{*}$, and the mapping $\Gamma:=\left(\Gamma_{0}, \Gamma_{1}\right)^{\top}: \operatorname{dom} S^{*} \rightarrow \mathcal{G} \times \mathcal{G}$ is surjective.

Recall that a boundary triple $\left\{\mathcal{G}, \Gamma_{0}, \Gamma_{1}\right\}$ for $S^{*}$ exists if and only if the defect numbers of $S$ coincide or, equivalently, $S$ admits self-adjoint extensions in $\mathfrak{H}$. Moreover, a boundary triple is not unique (except in the trivial case $S=S^{*}$ ). The following special observation will be used in Section 3: suppose that $\left\{\mathcal{G}, \Gamma_{0}, \Gamma_{1}\right\}$ is a boundary triple for $S^{*}$ and let $G$ be a bounded self-adjoint operator in $\mathcal{G}$; then $\left\{\mathcal{G}, \Gamma_{0}^{\prime}, \Gamma_{1}^{\prime}\right\}$, where

$$
\left(\begin{array}{l}
\Gamma_{0}^{\prime} \\
\Gamma_{1}^{\prime}
\end{array}\right)=\left(\begin{array}{cc}
I & G \\
0 & I
\end{array}\right)\left(\begin{array}{l}
\Gamma_{0} \\
\Gamma_{1}
\end{array}\right)
$$

is also a boundary triple for $S^{*}$. Recall also that $\operatorname{dom} S=\operatorname{ker} \Gamma_{0} \cap \operatorname{ker} \Gamma_{1}$ and that the mapping

$$
\Theta \mapsto A_{\Theta}:=S^{*} \uparrow\left\{f \in \operatorname{dom} S^{*}: \Gamma f=\left(\Gamma_{0} f, \Gamma_{1} f\right)^{\top} \in \Theta\right\}
$$


establishes a bijective correspondence between the closed linear subspaces (relations) in $\mathcal{G} \times \mathcal{G}$ and the closed linear extensions $A_{\Theta} \subset S^{*}$ of $S$. In the case when $\Theta$ is (the graph of) an operator, the closed extension $A_{\Theta}$ in (3) is given by

$$
A_{\Theta}=S^{*} \uparrow \operatorname{ker}\left(\Gamma_{1}-\Theta \Gamma_{0}\right) \text {. }
$$

It is important to note that the identity $\left(A_{\Theta}\right)^{*}=A_{\Theta^{*}}$ holds and hence $A_{\Theta}$ in (3)-(4) is selfadjoint in $\mathfrak{H}$ if and only if $\Theta$ is self-adjoint in $\mathcal{G}$. It follows, in particular, that the extension

$$
A_{0}=S^{*} \uparrow \operatorname{ker} \Gamma_{0}
$$

is self-adjoint. This extension often plays the role of a fixed extension within the family of self-adjoint extensions of $S$. We also mention that $\Theta$ in (3) is an unbounded operator if and only if the extensions $A_{0}$ and $A_{\Theta}$ are disjoint but not transversal, that is,

$$
S=A_{\Theta} \cap A_{0} \quad \text { and } \quad A_{\Theta} \widehat{+} A_{0} \subsetneq S^{*},
$$

where $\widehat{+}$ denotes the sum of subspaces. Note that this appears only in the case when $\mathcal{G}$ is infinite-dimensional, that is, the defect numbers of $S$ are both infinite.

The next theorem can be found in [39]. Very roughly speaking, it can be regarded as converse to the above considerations. Here, the idea is to start with boundary mappings defined on the domain of some operator $T$ that satisfy the abstract second Green's identity and some additional conditions, and to conclude that $T$ coincides with the adjoint of the restriction of $T$ to the intersection of the kernels of the boundary mappings. Theorem 2.2 will be used in the proof of Lemma 3.1.

Theorem 2.2. Let $T$ be a linear operator in $\mathfrak{H}$, let $\mathcal{G}$ be a Hilbert space and assume that $\Gamma_{0}, \Gamma_{1}: \operatorname{dom} T \rightarrow \mathcal{G}$ are linear mappings that satisfy the following conditions:

(i) there exists a self-adjoint restriction $A_{0}$ of $T$ in $\mathfrak{H}$ such that $\operatorname{dom} A_{0} \subset \operatorname{ker} \Gamma_{0}$;

(ii) $\operatorname{ran}\left(\Gamma_{0}, \Gamma_{1}\right)^{\top}=\mathcal{G} \times \mathcal{G}$;

(iii) for all $f, g \in \operatorname{dom} T$ the abstract Green's identity

$$
(T f, g)_{\mathfrak{H}}-(f, T g)_{\mathfrak{H}}=\left(\Gamma_{1} f, \Gamma_{0} g\right)_{\mathcal{G}}-\left(\Gamma_{0} f, \Gamma_{1} g\right)_{\mathcal{G}}
$$

holds.

Then $S:=T \uparrow\left(\operatorname{ker} \Gamma_{0} \cap \operatorname{ker} \Gamma_{1}\right)$ is a densely defined, closed, symmetric operator in $\mathfrak{H}$ such that $S^{*}=T$ and $\left\{\mathcal{G}, \Gamma_{0}, \Gamma_{1}\right\}$ is a boundary triple for $S^{*}$ with the property $A_{0}=S^{*} \uparrow \operatorname{ker} \Gamma_{0}$.

In the following, we assume that $S$ is a densely defined, closed, symmetric operator in $\mathfrak{H}$ and that $\left\{\mathcal{G}, \Gamma_{0}, \Gamma_{1}\right\}$ is a boundary triple for $S^{*}$. Let $A_{0}=S^{*} \uparrow \operatorname{ker} \Gamma_{0}$ be as in (5) and observe that the following direct sum decomposition of $\operatorname{dom} S^{*}$ is valid:

$$
\operatorname{dom} S^{*}=\operatorname{dom} A_{0} \dot{+} \operatorname{ker}\left(S^{*}-\lambda\right)=\operatorname{ker} \Gamma_{0} \dot{+} \operatorname{ker}\left(S^{*}-\lambda\right), \quad \lambda \in \rho\left(A_{0}\right) .
$$

It follows, in particular, that $\Gamma_{0} \uparrow \operatorname{ker}\left(S^{*}-\lambda\right)$ is a bijective operator from $\operatorname{ker}\left(S^{*}-\lambda\right)$ onto $\mathcal{G}$. The inverse is denoted by

$$
\gamma(\lambda)=\left(\Gamma_{0} \uparrow \operatorname{ker}\left(S^{*}-\lambda\right)\right)^{-1}, \quad \lambda \in \rho\left(A_{0}\right)
$$

when viewed as a function $\lambda \mapsto \gamma(\lambda)$ on $\rho\left(A_{0}\right)$, we call $\gamma$ the $\gamma$-field corresponding to the boundary triple $\left\{\mathcal{G}, \Gamma_{0}, \Gamma_{1}\right\}$. The Weyl function $M$ associated with $\left\{\mathcal{G}, \Gamma_{0}, \Gamma_{1}\right\}$ is defined by

$$
M(\lambda)=\Gamma_{1} \gamma(\lambda)=\Gamma_{1}\left(\Gamma_{0}\left\lceil\operatorname{ker}\left(S^{*}-\lambda\right)\right)^{-1}, \quad \lambda \in \rho\left(A_{0}\right) .\right.
$$

It can be shown that the values $M(\lambda)$ of the Weyl function $M$ are bounded, everywhere defined operators in $\mathcal{G}$, that $M$ is a holomorphic function on $\rho\left(A_{0}\right)$ with the properties $M(\lambda)=M(\bar{\lambda})^{*}$ and that $\operatorname{Im} M(\lambda)$ is uniformly positive for $\lambda \in \mathbb{C}^{+}$, i.e. $M$ is an operator-valued Nevanlinna or Riesz-Herglotz function that is uniformly strict; see [2]. 


\section{Schrödinger operators with $\delta$-interactions on hypersurfaces}

Let $\Omega_{i} \subset \mathbb{R}^{n}, n \geq 2$, be a bounded domain with $C^{\infty}$-smooth boundary $\mathcal{C}=\partial \Omega_{i}$ and let $\Omega_{e}=\mathbb{R}^{n} \backslash \bar{\Omega}_{i}$ be the corresponding exterior domain with the same $C^{\infty}$-smooth boundary $\partial \Omega_{e}=\mathcal{C}$. In the following, $\left.f_{i}\right|_{\mathcal{C}}$ and $\left.f_{e}\right|_{\mathcal{C}}$ denote the traces of functions in $\Omega_{i}$ and $\Omega_{e}$, respectively; if $\left.f_{i}\right|_{\mathcal{C}}=\left.f_{e}\right|_{\mathcal{C}}$, we also set $\left.f\right|_{\mathcal{C}}:=\left.f_{i}\right|_{\mathcal{C}}=\left.f_{e}\right|_{\mathcal{C}}$. Moreover, $\left.\partial_{\nu_{i}} f_{i}\right|_{\mathcal{C}}$ and $\left.\partial_{\nu_{e}} f_{e}\right|_{\mathcal{C}}$ denote the traces of their normal derivatives; here we agree that the normal vectors $\nu_{i}$ and $\nu_{e}$ point outwards of the domains, so that, $\nu_{i}=-\nu_{e}$.

In the following, let $\alpha \neq 0$ be a real constant and consider the Schrödinger operator with a $\delta$-interaction of strength $\alpha$ supported on $\mathcal{C}$ defined by

$$
\begin{aligned}
A_{\delta, \alpha} f & =-\Delta f \\
\operatorname{dom} A_{\delta, \alpha} & =\left\{f=\left(\begin{array}{c}
f_{i} \\
f_{e}
\end{array}\right) \in H^{2}\left(\Omega_{i}\right) \times H^{2}\left(\Omega_{e}\right), \begin{array}{c}
\left.f_{i}\right|_{\mathcal{C}}=\left.f_{e}\right|_{\mathcal{C}} \\
\left.\alpha f\right|_{\mathcal{C}}=\left.\partial_{\nu_{i}} f_{i}\right|_{\mathcal{C}}+\left.\partial_{\nu_{e}} f_{e}\right|_{\mathcal{C}}
\end{array}\right\} .
\end{aligned}
$$

According to [6, Theorem 3.5 and Theorem 3.6] the operator $A_{\delta, \alpha}$ is self-adjoint in $L^{2}\left(\mathbb{R}^{n}\right)$ and corresponds to the densely defined, closed sesquilinear form

$$
\begin{aligned}
\mathfrak{a}_{\delta, \alpha}[f, g] & =(\nabla f, \nabla g)_{\left(L^{2}\left(\mathbb{R}^{n}\right)\right)^{n}}-\alpha\left(\left.f\right|_{\mathcal{C}},\left.g\right|_{\mathcal{C}}\right)_{L^{2}(\mathcal{C})}, \\
\operatorname{dom} \mathfrak{a}_{\delta, \alpha} & =H^{1}\left(\mathbb{R}^{n}\right) .
\end{aligned}
$$

Observe that the normal derivatives of the functions in dom $A_{\delta, \alpha}$ may have a jump at the interface $\mathcal{C}$ or, more precisely, that $f \in \operatorname{dom} A_{\delta, \alpha}$ is contained in $H^{2}\left(\mathbb{R}^{n}\right)$ if and only if $\left.\partial_{\nu_{i}} f_{i}\right|_{\mathcal{C}}=-\left.\partial_{\nu_{e}} f_{e}\right|_{\mathcal{C}}$. We also recall that the essential spectrum of the operator $A_{\delta, \alpha}$ is $[0, \infty)$ and that the negative spectrum consists of a finite number of eigenvalues of finite multiplicity; see $[6,7]$. In the following, we fix some point $\eta$ such that

$$
\eta \in \rho\left(A_{\delta, \alpha}\right) \cap(-\infty, 0) .
$$

In Proposition 3.3 below, we specify a boundary triple $\left\{L^{2}(\mathcal{C}), \Gamma_{0}, \Gamma_{1}\right\}$ for the adjoint of the densely defined, closed, symmetric operator

$$
S f=-\Delta f, \quad \operatorname{dom} S=\left\{f \in H^{2}\left(\mathbb{R}^{n}\right):\left.f\right|_{\mathcal{C}}=0\right\},
$$

such that the free or unperturbed Schrödinger operator

$$
A_{\text {free }} f=-\Delta f, \quad \operatorname{dom} A_{\text {free }}=H^{2}\left(\mathbb{R}^{n}\right),
$$

corresponds to the kernel of the first boundary mapping $\Gamma_{0}$. Note that the operator $A_{\delta, \alpha}$ in (7) is a self-adjoint extension of $S$ and that the defect numbers $\operatorname{dim}\left(\operatorname{ran}(S \mp i)^{\perp}\right)$ are infinite. Hence, the abstract considerations in Section 2 ensure that there exists a self-adjoint operator or relation $\Theta_{\delta, \alpha}$ such that

$$
A_{\delta, \alpha}=S^{*} \uparrow \operatorname{ker}\left(\Gamma_{1}-\Theta_{\delta, \alpha} \Gamma_{0}\right)
$$

The parameter $\Theta_{\delta, \alpha}$ and further properties of the operator $A_{\delta, \alpha}$ will be discussed in Lemma 3.4 and Theorem 3.5 below.

Some further notations and preparatory results are required before Proposition 3.3 can be stated and proved. Consider the densely defined, closed, symmetric operators

$$
S_{i} f_{i}=-\Delta f_{i}, \quad \operatorname{dom} S_{i}=H_{0}^{2}\left(\Omega_{i}\right),
$$

and

$$
S_{e} f_{e}=-\Delta f_{e}, \quad \operatorname{dom} S_{e}=H_{0}^{2}\left(\Omega_{e}\right),
$$

in $L^{2}\left(\Omega_{i}\right)$ and $L^{2}\left(\Omega_{e}\right)$, respectively. Their adjoints are given by the maximal operators

$$
S_{i}^{*} f_{i}=-\Delta f_{i}, \quad \operatorname{dom} S_{i}^{*}=\left\{f_{i} \in L^{2}\left(\Omega_{i}\right):-\Delta f_{i} \in L^{2}\left(\Omega_{i}\right)\right\},
$$


and

$$
S_{e}^{*} f_{e}=-\Delta f_{e}, \quad \operatorname{dom} S_{e}^{*}=\left\{f_{e} \in L^{2}\left(\Omega_{e}\right):-\Delta f_{e} \in L^{2}\left(\Omega_{e}\right)\right\},
$$

where the expressions $-\Delta f_{i}$ and $-\Delta f_{e}$ are understood in the sense of distributions. It is important to note that $H^{2}\left(\Omega_{i}\right)$ and $H^{2}\left(\Omega_{e}\right)$ are proper subsets of the maximal domains $\operatorname{dom} S_{i}^{*}$ and $\operatorname{dom} S_{e}^{*}$, respectively, and that the symmetric operator $S$ in (9) is an infinite-dimensional extension of the orthogonal sum $S_{i} \oplus S_{e}$, which is also a symmetric operator in $L^{2}\left(\mathbb{R}^{n}\right)=$ $L^{2}\left(\Omega_{i}\right) \oplus L^{2}\left(\Omega_{e}\right)$. Recall from [27,40] that the trace maps admit continuous extensions to the maximal domains (equipped with the graph norms):

$$
\left.\operatorname{dom} S_{i}^{*} \ni f_{i} \mapsto f_{i}\right|_{\mathcal{C}} \in H^{-1 / 2}(\mathcal{C}),\left.\quad \operatorname{dom} S_{i}^{*} \ni f_{i} \mapsto \partial_{\nu_{i}} f_{i}\right|_{\mathcal{C}} \in H^{-3 / 2}(\mathcal{C}),
$$

and

$$
\left.\operatorname{dom} S_{e}^{*} \ni f_{e} \mapsto f_{e}\right|_{\mathcal{C}} \in H^{-1 / 2}(\mathcal{C}),\left.\quad \operatorname{dom} S_{e}^{*} \ni f_{e} \mapsto \partial_{\nu_{e}} f_{e}\right|_{\mathcal{C}} \in H^{-3 / 2}(\mathcal{C}) .
$$

Furthermore, consider the self-adjoint extensions $A_{i}^{D}$ and $A_{e}^{D}$ of $S_{i}$ and $S_{e}$, respectively, corresponding to Dirichlet boundary conditions on $\mathcal{C}$ :

$$
A_{i}^{D} f_{i}=-\Delta f_{i}, \quad \operatorname{dom} A_{i}^{D}=H_{0}^{1}\left(\Omega_{i}\right) \cap H^{2}\left(\Omega_{i}\right),
$$

and

$$
A_{e}^{D} f_{e}=-\Delta f_{e}, \quad \operatorname{dom} A_{e}^{D}=H_{0}^{1}\left(\Omega_{e}\right) \cap H^{2}\left(\Omega_{e}\right) .
$$

Since $A_{i}^{D}$ and $A_{e}^{D}$ are both non-negative, it is clear that $\eta$ in (8) belongs to $\rho\left(A_{i}^{D}\right) \cap \rho\left(A_{e}^{D}\right)$, and hence, we have the direct sum decompositions

$$
\operatorname{dom} S_{i}^{*}=\operatorname{dom} A_{i}^{D} \dot{+} \operatorname{ker}\left(S_{i}^{*}-\eta\right)=\left(H_{0}^{1}\left(\Omega_{i}\right) \cap H^{2}\left(\Omega_{i}\right)\right) \dot{+} \operatorname{ker}\left(S_{i}^{*}-\eta\right)
$$

and

$$
\operatorname{dom} S_{e}^{*}=\operatorname{dom} A_{e}^{D} \dot{+} \operatorname{ker}\left(S_{e}^{*}-\eta\right)=\left(H_{0}^{1}\left(\Omega_{e}\right) \cap H^{2}\left(\Omega_{e}\right)\right) \dot{+} \operatorname{ker}\left(S_{e}^{*}-\eta\right) .
$$

We agree to decompose functions $f_{i} \in \operatorname{dom} S_{i}^{*}$ and $f_{e} \in \operatorname{dom} S_{e}^{*}$ in the form

$$
f_{i}=f_{i}^{D}+f_{i}^{\eta} \quad \text { and } \quad f_{e}=f_{e}^{D}+f_{e}^{\eta}
$$

where $f_{i}^{D} \in \operatorname{dom} A_{i}^{D}, f_{i}^{\eta} \in \operatorname{ker}\left(S_{i}^{*}-\eta\right)$, and $f_{e}^{D} \in \operatorname{dom} A_{e}^{D}, f_{e}^{\eta} \in \operatorname{ker}\left(S_{e}^{*}-\eta\right)$.

In the following, we often make use of the operators

$$
\iota=\left(-\Delta_{\mathcal{C}}+I\right)^{\frac{1}{4}} \quad \text { and } \quad \iota^{-1}=\left(-\Delta_{\mathcal{C}}+I\right)^{-\frac{1}{4}}
$$

where $\Delta_{\mathcal{C}}$ denotes the Laplace-Beltrami operator on $\mathcal{C}$. Both mappings $\iota$ and $\iota^{-1}$ are regarded as isomorphisms

$$
\iota: H^{s}(\mathcal{C}) \rightarrow H^{s-\frac{1}{2}}(\mathcal{C}) \quad \text { and } \quad \iota^{-1}: H^{t}(\mathcal{C}) \rightarrow H^{t+\frac{1}{2}}(\mathcal{C})
$$

for $s, t \in \mathbb{R}$, and also as operators that establish the duality

$$
\left(\iota \varphi, \iota^{-1} \psi\right)_{L^{2}(\mathcal{C})}=\langle\varphi, \psi\rangle_{H^{1 / 2}(\mathcal{C}) \times H^{-1 / 2}(\mathcal{C})}
$$

for $\varphi \in H^{1 / 2}(\mathcal{C})$ and $\psi \in H^{-1 / 2}(\mathcal{C})$, when the spaces $H^{1 / 2}(\mathcal{C})$ and $H^{-1 / 2}(\mathcal{C})$ are equipped with the corresponding norms. Note also that $\iota^{-1}$ can be viewed as a bounded self-adjoint operator in $L^{2}(\mathcal{C})$ with $\operatorname{ran} \iota^{-1}=H^{1 / 2}(\mathcal{C})$ and that $\iota$ with domain $\operatorname{dom} \iota=H^{1 / 2}(\mathcal{C})$ is an unbounded self-adjoint operator in $L^{2}(\mathcal{C})$ with $0 \in \rho(\iota)$.

Now we have finally collected all necessary notation to state the first lemma. 
Lemma 3.1. Let $S$ be the densely defined, closed, symmetric operator in (9). Then the adjoint $S^{*}$ of $S$ is given by

$$
\begin{aligned}
S^{*} f & =-\Delta f \\
\operatorname{dom} S^{*} & =\left\{f=\left(\begin{array}{l}
f_{i} \\
f_{e}
\end{array}\right) \in \operatorname{dom} S_{i}^{*} \times \operatorname{dom} S_{e}^{*},\left.f_{i}\right|_{\mathcal{C}}=\left.f_{e}\right|_{\mathcal{C}}\right\} .
\end{aligned}
$$

Further, let

$$
\Upsilon_{0} f=\left.\iota^{-1} f\right|_{\mathcal{C}} \quad \text { and } \quad \Upsilon_{1} f=-\iota\left(\left.\partial_{\nu_{i}} f_{i}^{D}\right|_{\mathcal{C}}+\left.\partial_{\nu_{e}} f_{e}^{D}\right|_{\mathcal{C}}\right)
$$

for $f=\left(f_{i}, f_{e}\right)^{\top} \in \operatorname{dom} S^{*}$ and with $f_{i}^{D}, f_{e}^{D}$ as in (13). Then $\left\{L^{2}(\mathcal{C}), \Upsilon_{0}, \Upsilon_{1}\right\}$ is a boundary triple for $S^{*}$ with the property $A_{i}^{D} \oplus A_{e}^{D}=S^{*} \uparrow \operatorname{ker} \Upsilon_{0}$.

Proof. The assertions in Lemma 3.1 will be proved with the help of Theorem 2.2. To this end, we set

$$
\begin{aligned}
T f & =-\Delta f, \\
\operatorname{dom} T & =\left\{f=\left(\begin{array}{c}
f_{i} \\
f_{e}
\end{array}\right) \in \operatorname{dom} S_{i}^{*} \times \operatorname{dom} S_{e}^{*},\left.f_{i}\right|_{\mathcal{C}}=\left.f_{e}\right|_{\mathcal{C}}\right\},
\end{aligned}
$$

and consider the boundary mappings $\Upsilon_{0}, \Upsilon_{1}: \operatorname{dom} T \rightarrow L^{2}(\mathcal{C})$ in (15). First of all, we note that item (i) in Theorem 2.2 is satisfied with the self-adjoint operator $A_{0}=A_{i}^{D} \oplus A_{e}^{D}$ since for any function $f=\left(f_{i}, f_{e}\right)^{\top} \in \operatorname{dom}\left(A_{i}^{D} \oplus A_{e}^{D}\right) \subset H^{2}\left(\Omega_{i}\right) \times H^{2}\left(\Omega_{e}\right)$ one has $f_{i} \in \operatorname{dom} S_{i}^{*}$, $f_{e} \in \operatorname{dom} S_{e}^{*}$, and $\left.f_{i}\right|_{\mathcal{C}}=\left.f_{e}\right|_{\mathcal{C}}$. In order to see that the mapping

$$
\left(\begin{array}{l}
\Upsilon_{0} \\
\Upsilon_{1}
\end{array}\right): \operatorname{dom} T \rightarrow L^{2}(\mathcal{C}) \times L^{2}(\mathcal{C})
$$

is surjective, let $\varphi, \psi \in L^{2}(\mathcal{C})$. Since the Neumann trace map is surjective from $H^{2}\left(\Omega_{i}\right) \cap H_{0}^{1}\left(\Omega_{i}\right)$ onto $H^{1 / 2}(\mathcal{C})$ and from $H^{2}\left(\Omega_{e}\right) \cap H_{0}^{1}\left(\Omega_{e}\right)$ onto $H^{1 / 2}(\mathcal{C})$, there exist $f_{i}^{D} \in \operatorname{dom} A_{i}^{D}$ and $f_{e}^{D} \in$ $\operatorname{dom} A_{e}^{D}$ such that $\left.\partial_{\nu_{i}} f_{i}^{D}\right|_{\mathcal{C}}=\left.\partial_{\nu_{e}} f_{e}^{D}\right|_{\mathcal{C}}=-\frac{1}{2} \iota^{-1} \psi \in H^{1 / 2}(\mathcal{C})$. Next, we choose $f_{i}^{\eta} \in \operatorname{ker}\left(S_{i}^{*}-\eta\right)$ and $f_{e}^{\eta} \in \operatorname{ker}\left(S_{e}^{*}-\eta\right)$ such that $\left.f_{i}^{\eta}\right|_{\mathcal{C}}=\left.f_{e}^{\eta}\right|_{\mathcal{C}}=\iota \varphi \in H^{-1 / 2}(\mathcal{C})$, which is possible by the surjectivity of the trace map from the maximal domain onto $H^{-1 / 2}(\mathcal{C})$; cf. [27,40,41]. Now, it is easy to see that $f=\left(f_{i}^{D}+f_{i}^{\eta}, f_{e}^{D}+f_{e}^{\eta}\right)^{\top} \in \operatorname{dom} T$ satisfies

$$
\Upsilon_{0} f=\left.\iota^{-1} f\right|_{\mathcal{C}}=\varphi \quad \text { and } \quad \Upsilon_{1} f=-\iota\left(\left.\partial_{\nu_{i}} f_{i}^{D}\right|_{\mathcal{C}}+\left.\partial_{\nu_{e}} f_{e}^{D}\right|_{\mathcal{C}}\right)=\psi
$$

and hence the map (16) is onto. Next, we verify that the abstract second Green's identity

$$
(T f, g)_{L^{2}\left(\mathbb{R}^{n}\right)}-(f, T g)_{L^{2}\left(\mathbb{R}^{n}\right)}=\left(\Upsilon_{1} f, \Upsilon_{0} g\right)_{L^{2}(\mathcal{C})}-\left(\Upsilon_{0} f, \Upsilon_{1} g\right)_{L^{2}(\mathcal{C})}, \quad f, g \in \operatorname{dom} T
$$

holds. For this, it is useful to recall that Green's identity for $f_{i}=f_{i}^{D}+f_{i}^{\eta}$ and $g_{i}=g_{i}^{D}+g_{i}^{\eta}$ yields

$$
\begin{aligned}
\left(S_{i}^{*} f_{i}, g_{i}\right)_{L^{2}\left(\Omega_{i}\right)}- & \left(f_{i}, S_{i}^{*} g_{i}\right)_{L^{2}\left(\Omega_{i}\right)} \\
& =\left\langle\left. f_{i}\right|_{\mathcal{C}},\left.\partial_{\nu_{i}} g_{i}^{D}\right|_{\mathcal{C}}\right\rangle_{H^{-1 / 2}(\mathcal{C}) \times H^{1 / 2}(\mathcal{C})}-\left\langle\left.\partial_{\nu_{i}} f_{i}^{D}\right|_{\mathcal{C}},\left.g_{i}\right|_{\mathcal{C}}\right\rangle_{H^{1 / 2}(\mathcal{C}) \times H^{-1 / 2}(\mathcal{C})}
\end{aligned}
$$

and for $f_{e}=f_{e}^{D}+f_{e}^{\eta}$ and $g_{e}=g_{e}^{D}+g_{e}^{\eta}$ in the analogous form

$$
\begin{aligned}
\left(S_{e}^{*} f_{e}, g_{e}\right)_{L^{2}\left(\Omega_{e}\right)}- & \left(f_{e}, S_{e}^{*} g_{e}\right)_{L^{2}\left(\Omega_{e}\right)} \\
& =\left\langle\left. f_{e}\right|_{\mathcal{C}},\left.\partial_{\nu_{e}} g_{e}^{D}\right|_{\mathcal{C}}\right\rangle_{H^{-1 / 2}(\mathcal{C}) \times H^{1 / 2}(\mathcal{C})}-\left\langle\left.\partial_{\nu_{e}} f_{e}^{D}\right|_{\mathcal{C}},\left.g_{e}\right|_{\mathcal{C}}\right\rangle_{H^{1 / 2}(\mathcal{C}) \times H^{-1 / 2}(\mathcal{C})^{*}}
\end{aligned}
$$


Since $T$ is a restriction of the orthogonal sum $S_{i}^{*} \oplus S_{e}^{*}$ and $\left.f_{i}\right|_{\mathcal{C}}=\left.f_{e}\right|_{\mathcal{C}},\left.g_{i}\right|_{\mathcal{C}}=\left.g_{e}\right|_{\mathcal{C}}$ for $f, g \in \operatorname{dom} T$, we conclude from (18) and (19) that

$$
\begin{aligned}
(T f, g)_{L^{2}\left(\mathbb{R}^{n}\right)}-(f, T g)_{L^{2}\left(\mathbb{R}^{n}\right)} & \left(S_{i}^{*} f_{i}, g_{i}\right)_{L^{2}\left(\Omega_{i}\right)}-\left(f_{i}, S_{i}^{*} g_{i}\right)_{L^{2}\left(\Omega_{i}\right)}+\left(S_{e}^{*} f_{e}, g_{e}\right)_{L^{2}\left(\Omega_{e}\right)}-\left(f_{e}, S_{e}^{*} g_{e}\right)_{L^{2}\left(\Omega_{e}\right)} \\
= & \left\langle\left. f_{i}\right|_{\mathcal{C}},\left.\partial_{\nu_{i}} g_{i}^{D}\right|_{\mathcal{C}}\right\rangle_{H^{-1 / 2}(\mathcal{C}) \times H^{1 / 2}(\mathcal{C})}-\left\langle\left.\partial_{\nu_{i}} f_{i}^{D}\right|_{\mathcal{C}},\left.g_{i}\right|_{\mathcal{C}}\right\rangle_{H^{1 / 2}(\mathcal{C}) \times H^{-1 / 2}(\mathcal{C})} \\
& \quad+\left\langle\left. f_{e}\right|_{\mathcal{C}},\left.\partial_{\nu_{e}} g_{e}^{D}\right|_{\mathcal{C}}\right\rangle_{H^{-1 / 2}(\mathcal{C}) \times H^{1 / 2}(\mathcal{C})}-\left\langle\left.\partial_{\nu_{e}} f_{e}^{D}\right|_{\mathcal{C}},\left.g_{e}\right|_{\mathcal{C}}\right\rangle_{H^{1 / 2}(\mathcal{C}) \times H^{-1 / 2}(\mathcal{C})} \\
= & \left\langle\left. f\right|_{\mathcal{C}},\left.\partial_{\nu_{i}} g_{i}^{D}\right|_{\mathcal{C}}+\left.\partial_{\nu_{e}} g_{e}^{D}\right|_{\mathcal{C}}\right\rangle_{H^{-1 / 2}(\mathcal{C}) \times H^{1 / 2}(\mathcal{C})}-\left\langle\left.\partial_{\nu_{i}} f_{i}^{D}\right|_{\mathcal{C}}+\left.\partial_{\nu_{e}} f_{e}^{D}\right|_{\mathcal{C}},\left.g\right|_{\mathcal{C}}\right\rangle_{H^{1 / 2}(\mathcal{C}) \times H^{-1 / 2}(\mathcal{C})} \\
= & \left(\left.\iota^{-1} f\right|_{\mathcal{C}}, \iota\left(\left.\partial_{\nu_{i}} g_{i}^{D}\right|_{\mathcal{C}}+\left.\partial_{\nu_{e}} g_{e}^{D}\right|_{\mathcal{C}}\right)\right)_{L^{2}(\mathcal{C})}-\left(\iota\left(\left.\partial_{\nu_{i}} f_{i}^{D}\right|_{\mathcal{C}}+\left.\partial_{\nu_{e}} f_{e}^{D}\right|_{\mathcal{C}}\right),\left.\iota^{-1} g\right|_{\mathcal{C}}\right)_{L^{2}(\mathcal{C})} \\
= & \left(-\iota\left(\left.\partial_{\nu_{i}} f_{i}^{D}\right|_{\mathcal{C}}+\left.\partial_{\nu_{e}} f_{e}^{D}\right|_{\mathcal{C}}\right),\left.\iota^{-1} g\right|_{\mathcal{C}}\right)_{L^{2}(\mathcal{C})}-\left(\left.\iota^{-1} f\right|_{\mathcal{C}},-\iota\left(\left.\partial_{\nu_{i}} g_{i}^{D}\right|_{\mathcal{C}}+\left.\partial_{\nu_{e}} g_{e}^{D}\right|_{\mathcal{C}}\right)\right)_{L^{2}(\mathcal{C})} \\
= & \left(\Upsilon_{1} f, \Upsilon_{0} g\right)_{L^{2}(\mathcal{C})}-\left(\Upsilon_{0} f, \Upsilon_{1} g\right)_{L^{2}(\mathcal{C})}
\end{aligned}
$$

holds. Thus, (17) is shown and item (iii) in Theorem 2.2 is satisfied. Hence, Theorem 2.2 implies that the symmetric operator

$$
\widehat{S}:=T \uparrow\left(\operatorname{ker} \Upsilon_{0} \cap \operatorname{ker} \Upsilon_{1}\right)
$$

is densely defined, closed and its adjoint coincides with $T$. We show that $\widehat{S}$ coincides with the symmetric operator $S$ in (9). Note first that Theorem 2.2 also implies that

$$
A_{i}^{D} \oplus A_{e}^{D}=T \uparrow \operatorname{ker} \Upsilon_{0} .
$$

Both operators, $S$ and $\widehat{S}$, are restrictions of the operator in (21). We now let $f=\left(f_{i}, f_{e}\right)^{\top} \in$ $\operatorname{dom}\left(A_{i}^{D} \oplus A_{e}^{D}\right)=\operatorname{ker} \Upsilon_{0}$. For such $f$, we have

$$
\left.f \in \operatorname{ker} \Upsilon_{1} \Longleftrightarrow \partial_{\nu_{i}} f_{i}\right|_{\mathcal{C}}+\left.\partial_{\nu_{e}} f_{e}\right|_{\mathcal{C}}=0 \quad \Longleftrightarrow f \in H^{2}\left(\mathbb{R}^{n}\right) \Longleftrightarrow f \in \operatorname{dom} S .
$$

Thus, $\widehat{S}=S$. Now, the remaining statements in Lemma 3.1 follow immediately from Theorem 2.2.

Next, we specify the Weyl function $N$ and the $\gamma$-field $\zeta$ corresponding to the boundary triple $\left\{L^{2}(\mathcal{C}), \Upsilon_{0}, \Upsilon_{1}\right\}$ in Lemma 3.1. It is clear from the definition of $\Upsilon_{0}$ that the $\gamma$-field acts as follows:

$$
\zeta(\lambda): L^{2}(\mathcal{C}) \rightarrow L^{2}\left(\mathbb{R}^{n}\right), \quad \varphi \mapsto f_{\lambda}, \quad \lambda \in \rho\left(A_{i}^{D}\right) \cap \rho\left(A_{e}^{D}\right)=\mathbb{C} \backslash[0, \infty),
$$

where $f_{\lambda}=\left(f_{i, \lambda}, f_{e, \lambda}\right)^{\top} \in H^{2}\left(\Omega_{i}\right) \times H^{2}\left(\Omega_{e}\right)$ satisfies $-\Delta f_{i, \lambda}=\lambda f_{i, \lambda},-\Delta f_{e, \lambda}=\lambda f_{e, \lambda}$ and

$$
\left.f_{i, \lambda}\right|_{\mathcal{C}}=\left.f_{e, \lambda}\right|_{\mathcal{C}}=\iota \varphi
$$

In order to specify the Weyl function $N$, we recall the definition of the Dirichlet-to-Neumann maps $\mathcal{D}_{i}(\lambda)$ and $\mathcal{D}_{e}(\lambda)$ associated with the Laplacians on $\Omega_{i}$ and $\Omega_{e}$, respectively. Note first that for $\varphi, \psi \in H^{-1 / 2}(\mathcal{C})$ and $\lambda \in \rho\left(A_{i}^{D}\right)$ and $\mu \in \rho\left(A_{e}^{D}\right)$ the boundary value problems

$$
-\Delta f_{i}=\lambda f_{i},\left.\quad f_{i}\right|_{\mathcal{C}}=\varphi \quad \text { and } \quad-\Delta f_{e}=\mu f_{e},\left.\quad f_{e}\right|_{\mathcal{C}}=\psi
$$

admit unique solutions $f_{i, \lambda} \in \operatorname{dom} S_{i}^{*}$ and $f_{e, \mu} \in \operatorname{dom} S_{e}^{*}$. Hence, the operators

$$
\left.\mathcal{D}_{i,-1 / 2}(\lambda) f_{i, \lambda}\right|_{\mathcal{C}}=\left.\partial_{\nu_{i}} f_{i, \lambda}\right|_{\mathcal{C}}, \quad \operatorname{dom} \mathcal{D}_{i,-1 / 2}(\lambda)=H^{-1 / 2}(\mathcal{C}),
$$

and

$$
\left.\mathcal{D}_{e,-1 / 2}(\mu) f_{e, \mu}\right|_{\mathcal{C}}=\left.\partial_{\nu_{e}} f_{e, \lambda}\right|_{\mathcal{C}}, \quad \operatorname{dom} \mathcal{D}_{e,-1 / 2}(\mu)=H^{-1 / 2}(\mathcal{C}),
$$


are well defined, and map $H^{-1 / 2}(\mathcal{C})$ into $H^{-3 / 2}(\mathcal{C})$. We have used the index $-1 / 2$ in the definition of the Dirichlet-to-Neumann maps in (22) and (23) to indicate that their domain is $H^{-1 / 2}(\mathcal{C})$. For the following, it is important that the restrictions

$$
\left.\mathcal{D}_{i}(\lambda) f_{i, \lambda}\right|_{\mathcal{C}}=\left.\partial_{\nu_{i}} f_{i, \lambda}\right|_{\mathcal{C}}, \quad \operatorname{dom} \mathcal{D}_{i}(\lambda)=H^{1}(\mathcal{C})
$$

and

$$
\left.\mathcal{D}_{e}(\mu) f_{e, \mu}\right|_{\mathcal{C}}=\left.\partial_{\nu_{e}} f_{e, \mu}\right|_{\mathcal{C}}, \quad \operatorname{dom} \mathcal{D}_{e}(\mu)=H^{1}(\mathcal{C}),
$$

of $\mathcal{D}_{i,-1 / 2}(\lambda)$ and $\mathcal{D}_{e,-1 / 2}(\mu)$ to $H^{1}(\mathcal{C})$ are densely defined, closed, unbounded operators in $L^{2}(\mathcal{C})$ that satisfy

$$
\mathcal{D}_{i}(\lambda)^{*}=\mathcal{D}_{i}(\bar{\lambda}) \quad \text { and } \quad \mathcal{D}_{e}(\mu)^{*}=\mathcal{D}_{e}(\bar{\mu})
$$

for all $\lambda \in \rho\left(A_{i}^{D}\right)$ and for all $\mu \in \rho\left(A_{e}^{D}\right)$, respectively. For $\lambda \in \rho\left(A_{i}^{D}\right) \cap \rho\left(A_{e}^{D}\right)=\mathbb{C} \backslash[0, \infty)$, it is convenient to introduce the operators

$$
\mathcal{E}_{-1 / 2}(\lambda):=\mathcal{D}_{i,-1 / 2}(\lambda)+\mathcal{D}_{e,-1 / 2}(\lambda) \quad \text { and } \quad \mathcal{E}(\lambda):=\mathcal{D}_{i}(\lambda)+\mathcal{D}_{e}(\lambda)
$$

Furthermore, the restrictions of $\mathcal{D}_{i,-1 / 2}(\lambda)$ and $\mathcal{D}_{e,-1 / 2}(\mu)$ to $H^{3 / 2}(\mathcal{C})$ will be used. These restrictions are denoted by $\mathcal{D}_{i, 3 / 2}(\lambda)$ and $\mathcal{D}_{e, 3 / 2}(\mu)$, respectively; they map $H^{3 / 2}(\mathcal{C})$ into $H^{1 / 2}(\mathcal{C})$, and as above the index $3 / 2$ is used to indicate that their domain is $H^{3 / 2}(\mathcal{C})$.

Lemma 3.2. Let $S$ be the symmetric operator in (9), let $\left\{L^{2}(\mathcal{C}), \Upsilon_{0}, \Upsilon_{1}\right\}$ be the boundary triple for $S^{*}$ in Lemma 3.1 and fix $\eta$ as in (8). For $\lambda \in \rho\left(A_{i}^{D}\right) \cap \rho\left(A_{e}^{D}\right)=\mathbb{C} \backslash[0, \infty)$ the operators $\mathcal{E}_{-1 / 2}(\lambda)$ in (24) have the property

$$
\operatorname{ran}\left(\mathcal{E}_{-1 / 2}(\lambda)-\mathcal{E}_{-1 / 2}(\eta)\right) \subset H^{1 / 2}(\mathcal{C})
$$

and the Weyl function corresponding to the boundary triple $\left\{L^{2}(\mathcal{C}), \Upsilon_{0}, \Upsilon_{1}\right\}$ is given by

$$
N(\lambda)=-\iota\left(\mathcal{E}_{-1 / 2}(\lambda)-\mathcal{E}_{-1 / 2}(\eta)\right) \iota, \quad \lambda \in \mathbb{C} \backslash[0, \infty) .
$$

Proof. Let $\lambda \in \rho\left(A_{i}^{D}\right) \cap \rho\left(A_{e}^{D}\right)$ and let $f_{\lambda}=\left(f_{i, \lambda}, f_{e, \lambda}\right) \in \operatorname{ker}\left(S^{*}-\lambda\right)$. Then $\left.f_{i, \lambda}\right|_{\mathcal{C}}=\left.f_{e, \lambda}\right|_{\mathcal{C}}$ and according to (11)-(13) we have

$$
f_{i, \lambda}=f_{i, \lambda}^{D}+f_{i, \lambda}^{\eta} \quad \text { and } \quad f_{e, \lambda}=f_{e, \lambda}^{D}+f_{e, \lambda}^{\eta},
$$

where $f_{i, \lambda}^{D} \in \operatorname{dom} A_{i}^{D}, f_{i, \lambda}^{\eta} \in \operatorname{ker}\left(S_{i}^{*}-\eta\right), f_{e, \lambda}^{D} \in \operatorname{dom} A_{e}^{D}$ and $f_{e, \lambda}^{\eta} \in \operatorname{ker}\left(S_{e}^{*}-\eta\right)$. Hence, it follows with the help of $\left.f_{i, \lambda}\right|_{\mathcal{C}}=\left.f_{i, \lambda}^{\eta}\right|_{\mathcal{C}}$ and $\left.f_{e, \lambda}\right|_{\mathcal{C}}=\left.f_{e, \lambda}^{\eta}\right|_{\mathcal{C}}$, and the definition of the Dirichletto-Neumann maps that

$$
\begin{aligned}
\left(\mathcal{E}_{-1 / 2}(\lambda)\right. & \left.-\mathcal{E}_{-1 / 2}(\eta)\right) \iota \Upsilon_{0} f_{\lambda} \\
= & \left.\left(\mathcal{D}_{i,-1 / 2}(\lambda)-\mathcal{D}_{i,-1 / 2}(\eta)+\mathcal{D}_{e,-1 / 2}(\lambda)-\mathcal{D}_{e,-1 / 2}(\eta)\right) f_{\lambda}\right|_{\mathcal{C}} \\
= & \left.\mathcal{D}_{i,-1 / 2}(\lambda) f_{i, \lambda}\right|_{\mathcal{C}}-\left.\mathcal{D}_{i,-1 / 2}(\eta) f_{i, \lambda}^{\eta}\right|_{\mathcal{C}}+\left.\mathcal{D}_{e,-1 / 2}(\lambda) f_{e, \lambda}\right|_{\mathcal{C}}-\left.\mathcal{D}_{e,-1 / 2}(\eta) f_{e, \lambda}^{\eta}\right|_{\mathcal{C}} \\
= & \left.\partial_{\nu_{i}} f_{i, \lambda}\right|_{\mathcal{C}}-\left.\partial_{\nu_{i}} f_{i, \lambda}^{\eta}\right|_{\mathcal{C}}+\left.\partial_{\nu_{e}} f_{e, \lambda}\right|_{\mathcal{C}}-\left.\partial_{\nu_{e}} f_{e, \lambda}^{\eta}\right|_{\mathcal{C}} \\
= & \left.\partial_{\nu_{i}}\left(f_{i, \lambda}-f_{i, \lambda}^{\eta}\right)\right|_{\mathcal{C}}+\left.\partial_{\nu_{e}}\left(f_{e, \lambda}-f_{e, \lambda}^{\eta}\right)\right|_{\mathcal{C}} \\
= & \left.\partial_{\nu_{i}} f_{i, \lambda}^{D}\right|_{\mathcal{C}}+\left.\partial_{\nu_{e}} f_{e, \lambda}^{D}\right|_{\mathcal{C}}
\end{aligned}
$$

and hence

$$
-\iota\left(\mathcal{E}_{-1 / 2}(\lambda)-\mathcal{E}_{-1 / 2}(\eta)\right) \iota \Upsilon_{0} f_{\lambda}=-\iota\left(\left.\partial_{\nu_{i}} f_{i, \lambda}^{D}\right|_{\mathcal{C}}+\left.\partial_{\nu_{e}} f_{e, \lambda}^{D}\right|_{\mathcal{C}}\right)=\Upsilon_{1} f_{\lambda}
$$

Also, the inclusion (25) follows from (26) since $f_{i, \lambda}^{D} \in H^{2}\left(\Omega_{i}\right)$ and $f_{e, \lambda}^{D} \in H^{2}\left(\Omega_{e}\right)$, and hence, $\left.\partial_{\nu_{i}} f_{i, \lambda}^{D}\right|_{\mathcal{C}}+\left.\partial_{\nu_{e}} f_{e, \lambda}^{D}\right|_{\mathcal{C}} \in H^{1 / 2}(\mathcal{C})$ in (26), and for any

$$
\varphi \in \operatorname{dom} \mathcal{E}_{-1 / 2}(\lambda)=\operatorname{dom} \mathcal{E}_{-1 / 2}(\eta)=H^{-1 / 2}(\mathcal{C}),
$$


there exists $f_{\lambda}=\left(f_{i, \lambda}, f_{e, \lambda}\right)^{\top} \in \operatorname{ker}\left(S^{*}-\lambda\right)$ and $f_{\eta}=\left(f_{i, \eta}, f_{e, \eta}\right) \in \operatorname{ker}\left(S^{*}-\eta\right)$ such that

$$
\left.f_{i, \lambda}\right|_{\mathcal{C}}=\left.f_{e, \lambda}\right|_{\mathcal{C}}=\varphi=\left.f_{i, \eta}\right|_{\mathcal{C}}=\left.f_{e, \eta}\right|_{\mathcal{C}}
$$

In the following proposition, we provide a boundary triple for $S^{*}$ such that the operator $A_{\text {free }}$ corresponds to the first boundary mapping. For this, we modify the boundary triple in Lemma 3.1 in a suitable manner.

Proposition 3.3. Let $S$ be the densely defined, closed, symmetric operator in (9) with adjoint $S^{*}$ in $(14)$, and let $\mathfrak{E}(\eta)=\iota \mathcal{E}(\eta) \iota$. Then, $\mathfrak{E}(\eta)^{-1}$ is a bounded self-adjoint operator in $L^{2}(\mathcal{C})$ and $\left\{L^{2}(\mathcal{C}), \Gamma_{0}, \Gamma_{1}\right\}$, where

$$
\Gamma_{0} f=\left.\iota^{-1} f\right|_{\mathcal{C}}+\mathfrak{E}(\eta)^{-1} \iota\left(\left.\partial_{\nu_{i}} f_{i}^{D}\right|_{\mathcal{C}}+\left.\partial_{\nu_{e}} f_{e}^{D}\right|_{\mathcal{C}}\right) \text { and } \Gamma_{1} f=-\iota\left(\left.\partial_{\nu_{i}} f_{i}^{D}\right|_{\mathcal{C}}+\left.\partial_{\nu_{e}} f_{e}^{D}\right|_{\mathcal{C}}\right),
$$

is a boundary triple for $S^{*}$ with the property $A_{\text {free }}=S^{*} \uparrow \operatorname{ker} \Gamma_{0}$. For $\lambda \in \mathbb{C} \backslash[0, \infty)$ the Weyl function corresponding to $\left\{L^{2}(\mathcal{C}), \Gamma_{0}, \Gamma_{1}\right\}$ is given by

$$
M(\lambda)=-\iota\left(\mathcal{E}_{-1 / 2}(\lambda)-\mathcal{E}_{-1 / 2}(\eta)\right) \iota\left(I+\mathfrak{E}(\eta)^{-1} \iota\left(\mathcal{E}_{-1 / 2}(\lambda)-\mathcal{E}_{-1 / 2}(\eta)\right) \iota\right) .
$$

Proof. First, we show that $\mathfrak{E}(\eta)^{-1}=\iota^{-1} \mathcal{E}(\eta)^{-1} \iota^{-1}$ is a bounded self-adjoint operator in $L^{2}(\mathcal{C})$. Observe that $\mathcal{E}(\eta)=\mathcal{D}_{i}(\eta)+\mathcal{D}_{e}(\eta)$ is injective. In fact, we assume that $\mathcal{E}(\eta) \varphi=0$ for some $\varphi \in H^{1}(\mathcal{C}), \varphi \neq 0$. Then, there exists $f_{\eta}=\left(f_{i}^{\eta}, f_{e}^{\eta}\right)^{\top} \in \operatorname{ker}\left(S^{*}-\eta\right)$ such that $\left.f_{\eta}\right|_{\mathcal{C}}=\varphi$ and hence

$$
0=\mathcal{E}(\eta) \varphi=\left.\mathcal{E}(\eta) f_{\eta}\right|_{\mathcal{C}}=\left.\mathcal{D}_{i}(\eta) f_{i}^{\eta}\right|_{\mathcal{C}}+\left.\mathcal{D}_{e}(\eta) f_{e}^{\eta}\right|_{\mathcal{C}}=\left.\partial_{\nu_{i}} f_{i}^{\eta}\right|_{\mathcal{C}}+\left.\partial_{\nu_{e}} f_{e}^{\eta}\right|_{\mathcal{C}}
$$

Together with $\left.f_{i}^{\eta}\right|_{\mathcal{C}}=\left.f_{e}^{\eta}\right|_{\mathcal{C}}$, this implies that $f_{\eta} \in \operatorname{dom} A_{\text {free }}$ and hence $\operatorname{ker}\left(A_{\text {free }}-\eta\right) \neq\{0\}$. This is impossible, as $\eta<0$. Thus $\mathcal{E}(\eta)$ is injective. It follows from [6, Proposition 3.2 (iii)] that $\mathcal{E}(\eta)$ is surjective. Hence, $\mathcal{E}(\eta)^{-1}$ is a bounded self-adjoint operator in $L^{2}(\mathcal{C})$. Since $\iota^{-1}$ is also a bounded self-adjoint operator in $L^{2}(\mathcal{C})$, it is clear that $\mathfrak{E}(\eta)^{-1}=\iota^{-1} \mathcal{E}(\eta)^{-1} \iota^{-1}$ is a bounded self-adjoint operator in $L^{2}(\mathcal{C})$.

Now, let $\left\{L^{2}(\mathcal{C}), \Upsilon_{0}, \Upsilon_{1}\right\}$ be the boundary triple in Lemma 3.1. Note that the boundary mappings $\Gamma_{0}$ and $\Gamma_{1}$ in (27) satisfy:

$$
\left(\begin{array}{l}
\Gamma_{0} \\
\Gamma_{1}
\end{array}\right)=\left(\begin{array}{cc}
I & -\mathfrak{E}(\eta)^{-1} \\
0 & I
\end{array}\right)\left(\begin{array}{l}
\Upsilon_{0} \\
\Upsilon_{1}
\end{array}\right)
$$

Hence, it follows that $\left\{L^{2}(\mathcal{C}), \Gamma_{0}, \Gamma_{1}\right\}$ is a boundary triple for $S^{*}$; see (2) in Section 2. Again, we let $N$ denote the Weyl function corresponding to the boundary triple $\left\{L^{2}(\mathcal{C}), \Upsilon_{0}, \Upsilon_{1}\right\}$. It is not difficult to see that the Weyl function corresponding to $\left\{L^{2}(\mathcal{C}), \Gamma_{0}, \Gamma_{1}\right\}$ is given by

$$
M(\lambda)=N(\lambda)\left(I-\mathfrak{E}(\eta)^{-1} N(\lambda)\right)^{-1}, \quad \lambda \in \mathbb{C} \backslash[0, \infty) .
$$

Hence, the form of the Weyl function $M$ follows from Lemma 3.2.

It remains to be shown that $A_{\text {free }}=S^{*} \uparrow \operatorname{ker} \Gamma_{0}$ holds. Assume that for some $f \in \operatorname{dom} S^{*}$ we have

$$
\left.\iota^{-1} f\right|_{\mathcal{C}}+\mathfrak{E}(\eta)^{-1} \iota\left(\left.\partial_{\nu_{i}} f_{i}^{D}\right|_{\mathcal{C}}+\left.\partial_{\nu_{e}} f_{e}^{D}\right|_{\mathcal{C}}\right)=0 .
$$

As $\operatorname{ker} \mathcal{E}_{-1 / 2}(\eta)=\{0\}$ (this can be seen as in (28)), this is equivalent to

$$
\left.\mathcal{E}_{-1 / 2}(\eta) f\right|_{\mathcal{C}}+\left(\left.\partial_{\nu_{i}} f_{i}^{D}\right|_{\mathcal{C}}+\left.\partial_{\nu_{e}} f_{e}^{D}\right|_{\mathcal{C}}\right)=0
$$

Furthermore, since

$$
\left.\mathcal{E}_{-1 / 2}(\eta) f\right|_{\mathcal{C}}=\left.\mathcal{D}_{i,-1 / 2}(\eta) f_{i}^{\eta}\right|_{\mathcal{C}}+\left.\mathcal{D}_{e,-1 / 2}(\eta) f_{e}^{\eta}\right|_{\mathcal{C}}=\left.\partial_{\nu_{i}} f_{i}^{\eta}\right|_{\mathcal{C}}+\left.\partial_{\nu_{e}} f_{e}^{\eta}\right|_{\mathcal{C}}
$$

holds for $f$ decomposed as in (13), we conclude that (29) is equivalent to

$$
\left.\partial_{\nu_{i}} f_{i}^{\eta}\right|_{\mathcal{C}}+\left.\partial_{\nu_{e}} f_{e}^{\eta}\right|_{\mathcal{C}}+\left.\partial_{\nu_{i}} f_{i}^{D}\right|_{\mathcal{C}}+\left.\partial_{\nu_{e}} f_{e}^{D}\right|_{\mathcal{C}}=0
$$


which in turn is equivalent to

$$
\left.\partial_{\nu_{i}} f_{i}\right|_{\mathcal{C}}+\left.\partial_{\nu_{e}} f_{e}\right|_{\mathcal{C}}=0
$$

Therefore, $f \in \operatorname{ker} \Gamma_{0}$ if and only if $f \in \operatorname{dom} A_{\text {free }}$.

Our next goal is to identify the self-adjoint parameter $\Theta_{\delta, \alpha}$ such that (10) holds with the boundary triple in Proposition 3.3.

Lemma 3.4. Let $S$ be the densely defined, closed, symmetric operator in (9) with adjoint $S^{*}$ in (14), and let $\left\{L^{2}(\mathcal{C}), \Gamma_{0}, \Gamma_{1}\right\}$ be the boundary triple in Proposition 3.3. Then

$$
\Theta_{\delta, \alpha}=\iota\left(\mathcal{D}_{i, 3 / 2}(\eta)+\mathcal{D}_{e, 3 / 2}(\eta)-\alpha\right) \iota\left(I-\mathfrak{E}(\eta)^{-1} \iota\left(\mathcal{D}_{i, 3 / 2}(\eta)+\mathcal{D}_{e, 3 / 2}(\eta)-\alpha\right) \iota\right)^{-1}
$$

is an unbounded self-adjoint operator in $L^{2}(\mathcal{C})$ such that the Schrödinger operator $A_{\delta, \alpha}$ in (7) corresponds to $\Theta_{\delta, \alpha}$, that is,

$$
A_{\delta, \alpha}=S^{*} \uparrow \operatorname{ker}\left(\Gamma_{1}-\Theta_{\delta, \alpha} \Gamma_{0}\right) .
$$

Proof. We make use of the fact that the boundary triple $\left\{L^{2}(\mathcal{C}), \Upsilon_{0}, \Upsilon_{1}\right\}$ in Lemma 3.1 and the boundary triple $\left\{L^{2}(\mathcal{C}), \Gamma_{0}, \Gamma_{1}\right\}$ in Proposition 3.3 are related via

$$
\Gamma_{0}=\Upsilon_{0}-\mathfrak{E}(\eta)^{-1} \Upsilon_{1} \quad \text { and } \quad \Gamma_{1}=\Upsilon_{1},
$$

and we also make use of the operator

$$
\Lambda_{\delta, \alpha}=\iota\left(\mathcal{D}_{i, 3 / 2}(\eta)+\mathcal{D}_{e, 3 / 2}(\eta)-\alpha\right) \iota, \quad \operatorname{dom} \Lambda_{\delta, \alpha}=H^{2}(\mathcal{C}) .
$$

Our first task is to show that

$$
A_{\delta, \alpha}=S^{*} \uparrow \operatorname{ker}\left(\Upsilon_{1}-\Lambda_{\delta, \alpha} \Upsilon_{0}\right)
$$

holds. In fact, $f \in \operatorname{ker}\left(\Upsilon_{1}-\Lambda_{\delta, \alpha} \Upsilon_{0}\right)$ if and only if $f \in \operatorname{dom} S^{*}$ and

$$
\left.\iota\left(\mathcal{D}_{i, 3 / 2}(\eta)+\mathcal{D}_{e, 3 / 2}(\eta)-\alpha\right) f\right|_{\mathcal{C}}=-\iota\left(\left.\partial_{\nu_{i}} f_{i}^{D}\right|_{\mathcal{C}}+\left.\partial_{\nu_{e}} f_{e}^{D}\right|_{\mathcal{C}}\right),
$$

where $\left.f\right|_{\mathcal{C}} \in \operatorname{dom} \mathcal{D}_{i, 3 / 2}(\eta)=\operatorname{dom} \mathcal{D}_{e, 3 / 2}(\eta)=H^{3 / 2}(\mathcal{C})$, together with elliptic regularity, also implies that $f=\left(f_{i}, f_{e}\right)^{\top}$ with $f_{i} \in H^{2}\left(\Omega_{i}\right)$ and $f_{e} \in H^{2}\left(\Omega_{e}\right)$. With $f$ decomposed as in (13) we have

$$
\left.\left(\mathcal{D}_{i, 3 / 2}(\eta)+\mathcal{D}_{e, 3 / 2}(\eta)-\alpha\right) f\right|_{\mathcal{C}}=\left.\partial_{\nu_{i}} f_{i}^{\eta}\right|_{\mathcal{C}}+\left.\partial_{\nu_{e}} f_{e}^{\eta}\right|_{\mathcal{C}}-\left.\alpha f\right|_{\mathcal{C}} .
$$

Therefore, $f \in \operatorname{ker}\left(\Upsilon_{1}-\Lambda_{\delta, \alpha} \Upsilon_{0}\right)$ if and only if $f=\left(f_{i}, f_{e}\right)^{\top} \in \operatorname{dom} S^{*}$ with $f_{i} \in H^{2}\left(\Omega_{i}\right)$ and $f_{e} \in H^{2}\left(\Omega_{e}\right)$ and

$$
\left.\partial_{\nu_{i}} f_{i}^{\eta}\right|_{\mathcal{C}}+\left.\partial_{\nu_{e}} f_{e}^{\eta}\right|_{\mathcal{C}}-\left.\alpha f\right|_{\mathcal{C}}=-\left(\left.\partial_{\nu_{i}} f_{i}^{D}\right|_{\mathcal{C}}+\left.\partial_{\nu_{e}} f_{e}^{D}\right|_{\mathcal{C}}\right),
$$

and the latter can be rewritten in the form

$$
\left.\partial_{\nu_{i}} f_{i}\right|_{\mathcal{C}}+\left.\partial_{\nu_{e}} f_{e}\right|_{\mathcal{C}}=\left.\alpha f\right|_{\mathcal{C}}
$$

We have shown (33), and as $A_{\delta, \alpha}$ is a self-adjoint operator in $L^{2}\left(\mathbb{R}^{n}\right)$, it follows that $\Lambda_{\delta, \alpha}$ in (32) is an unbounded self-adjoint operator in $L^{2}(\mathcal{C})$.

Next, we consider the operator

$$
\Theta_{\delta, \alpha}=\Lambda_{\delta, \alpha}\left(I-\mathfrak{E}(\eta)^{-1} \Lambda_{\delta, \alpha}\right)^{-1}
$$

on its natural domain; note that $\operatorname{ker}\left(I-\mathfrak{E}(\eta)^{-1} \Lambda_{\delta, \alpha}\right)=\{0\}$ as otherwise $\mathfrak{E}(\eta) \varphi=\Lambda_{\delta, \alpha} \varphi$ for some non-trivial $\varphi \in H^{2}(\mathcal{C})$, which is a contradiction to $\alpha \neq 0$. Now, we assume that 
$f \in \operatorname{ker}\left(\Gamma_{1}-\Theta_{\delta, \alpha} \Gamma_{0}\right)$. Then, (31) and (34) yield

$$
\begin{aligned}
\Upsilon_{1} f-\Lambda_{\delta, \alpha} \Upsilon_{0} f & =\Gamma_{1} f-\Lambda_{\delta, \alpha}\left(\Gamma_{0}+\mathfrak{E}(\eta)^{-1} \Upsilon_{1}\right) f \\
& =\Gamma_{1} f-\Lambda_{\delta, \alpha}\left(\Gamma_{0}+\mathfrak{E}(\eta)^{-1} \Gamma_{1}\right) f \\
& =\Gamma_{1} f-\Lambda_{\delta, \alpha}\left(\Gamma_{0}+\mathfrak{E}(\eta)^{-1} \Theta_{\delta, \alpha} \Gamma_{0}\right) f \\
& =\Gamma_{1} f-\Lambda_{\delta, \alpha}\left(I+\mathfrak{E}(\eta)^{-1} \Lambda_{\delta, \alpha}\left(I-\mathfrak{E}(\eta)^{-1} \Lambda_{\delta, \alpha}\right)^{-1}\right) \Gamma_{0} f \\
& =\Gamma_{1} f-\Lambda_{\delta, \alpha}\left(I-\mathfrak{E}(\eta)^{-1} \Lambda_{\delta, \alpha}\right)^{-1} \Gamma_{0} f \\
& =\Gamma_{1} f-\Theta_{\delta, \alpha} \Gamma_{0} f \\
& =0
\end{aligned}
$$

and hence $f \in \operatorname{ker}\left(\Upsilon_{1}-\Lambda_{\delta, \alpha} \Upsilon_{0}\right)$. The converse inclusion is shown in the same way and therefore

$$
\operatorname{ker}\left(\Gamma_{1}-\Theta_{\delta, \alpha} \Gamma_{0}\right)=\operatorname{ker}\left(\Upsilon_{1}-\Lambda_{\delta, \alpha} \Upsilon_{0}\right)
$$

and thus the extensions

$$
S^{*} \uparrow \operatorname{ker}\left(\Gamma_{1}-\Theta_{\delta, \alpha} \Gamma_{0}\right) \quad \text { and } \quad S^{*} \uparrow \operatorname{ker}\left(\Upsilon_{1}-\Lambda_{\delta, \alpha} \Upsilon_{0}\right),
$$

coincide. Therefore, (33) implies (30). Since $A_{\delta, \alpha}$ is self-adjoint in $L^{2}\left(\mathbb{R}^{n}\right)$, it also follows from (30) that $\Theta_{\delta, \alpha}$ is self-adjoint in $L^{2}(\mathcal{C})$. Moreover, as $S$ in (9) coincides with the intersection of $A_{\text {free }}$ and $A_{\delta, \alpha}$, that is, $A_{\text {free }}$ and $A_{\delta, \alpha}$ are disjoint, and since $A_{\text {free }}$ and $A_{\delta, \alpha}$ are not transversal, one concludes that $\Theta_{\delta, \alpha}$ is an unbounded operator in $L^{2}(\mathcal{C})$; cf. (6).

We are now able to obtain some immediate and important consequences from the previous considerations, well-known results for boundary triples and Weyl functions $[3,4]$ and the resolvent estimates in $[6,42]$.

Theorem 3.5. Let $S$ be the densely defined, closed, symmetric operator in (9) with adjoint $S^{*}$ in (14), let $\left\{L^{2}(\mathcal{C}), \Gamma_{0}, \Gamma_{1}\right\}$ be the boundary triple in Proposition 3.3 with

$$
A_{\text {free }}=S^{*} \uparrow \operatorname{ker} \Gamma_{0}
$$

and let $\gamma$ and $M$ be the $\gamma$-field and Weyl function corresponding to $\left\{L^{2}(\mathcal{C}), \Gamma_{0}, \Gamma_{1}\right\}$. Furthermore, let $\Theta_{\delta, \alpha}$ be as in Lemma 3.4 so that

$$
A_{\delta, \alpha}=S^{*} \uparrow \operatorname{ker}\left(\Gamma_{1}-\Theta_{\delta, \alpha} \Gamma_{0}\right) .
$$

Then, the following assertions hold for all $\lambda \notin[0, \infty)$ :

(i) $\lambda \in \sigma_{p}\left(A_{\delta, \alpha}\right)$ if and only if $0 \in \sigma_{p}\left(\Theta_{\delta, \alpha}-M(\lambda)\right)$;

(ii) $\lambda \in \rho\left(A_{\delta, \alpha}\right)$ if and only if $0 \in \rho\left(\Theta_{\delta, \alpha}-M(\lambda)\right)$;

(iii) for all $\lambda \in \rho\left(A_{\delta, \alpha}\right)$ the resolvent formula

$$
\left(A_{\delta, \alpha}-\lambda\right)^{-1}-\left(A_{\text {free }}-\lambda\right)^{-1}=\gamma(\lambda)\left(\Theta_{\delta, \alpha}-M(\lambda)\right)^{-1} \gamma(\bar{\lambda})^{*}
$$

is valid, and the resolvent difference of $A_{\delta, \alpha}$ and $A_{\text {free }}$ belongs to the Schatten-von Neumann ideal $\mathfrak{S}_{p}\left(L^{2}\left(\mathbb{R}^{n}\right)\right)$ for all $p>\frac{n-1}{3}$;

(iv) for all $\xi \in \rho\left(\Theta_{\delta, \alpha}\right)$ the operator $\left(\Theta_{\delta, \alpha}-\xi\right)^{-1}$ belongs to the Schatten-von Neumann ideal $\mathfrak{S}_{p}\left(L^{2}(\mathcal{C})\right)$ for all $p>\frac{n-1}{3}$. 


\section{Acknowledgements}

J. Behrndt and V. Lotoreichik gratefully acknowledge financial support by the Austrian Science Fund (FWF): Project P 25162-N26. J. Behrndt also wishes to thank Professor Igor Popov for the pleasant and fruitful research stay at the ITMO University in St. Petersburg in September 2015. V. Lotoreichik was also supported by the Czech Science Foundation (GAČR) under the project 14-06818S.

\section{References}

[1] Brüning J., Geyler V., Pankrashkin K. Spectra of self-adjoint extensions and applications to solvable Schrödinger operators. Rev. Math. Phys., 2008, 20, P. 1-70.

[2] Derkach V. A., Hassi S., Malamud M. M., de Snoo H. Boundary relations and their Weyl families. Trans. Amer. Math. Soc., 2006, 358, P. 5351-5400.

[3] Derkach V.A., Malamud M.M. Generalized resolvents and the boundary value problems for Hermitian operators with gaps. J. Funct. Anal., 1991, 95, P. 1-95.

[4] Derkach V.A., Malamud M. M. The extension theory of Hermitian operators and the moment problem. $J$. Math. Sci., 1995, 73, P. 141-242.

[5] Gorbachuk V.I., Gorbachuk M. L. Boundary Value Problems for Operator Differential Equations, Kluwer Academic Publ., Dordrecht, 1991.

[6] Behrndt J., Langer M., Lotoreichik V. Schrödinger operators with $\delta$ and $\delta^{\prime}$-potentials supported on hypersurfaces. Ann. Henri Poincaré, 2013, 14, P. 385-423.

[7] Brasche J.F., Exner P., Kuperin Yu. A., Šeba P. Schrödinger operators with singular interactions. J. Math. Anal. Appl., 1994, 184, P. 112-139.

[8] Albeverio S., Gesztesy F., Hoegh-Krohn R., Holden H. Solvable Models in Quantum Mechanics. With an appendix by Pavel Exner. AMS Chelsea Publishing, 2005.

[9] Exner P., Kovařik H. Quantum Waveguides, Theoretical and Mathematical Physics, Springer, 2015.

[10] Exner P. Leaky quantum graphs: a review. Proc. Symp. Pure Math., 2008, 77, P. 523-564.

[11] Albeverio S., Kostenko A., Malamud M., Neidhardt H. Spherical Schrödinger operators with $\delta$-type interactions. J. Math. Phys., 2013, 54, 052103, 24 pp.

[12] Behrndt J., Exner P., Lotoreichik V. Schrödinger operators with $\delta$ and $\delta^{\prime}$ interactions on Lipschitz surfaces and chromatic numbers of associated partitions. Rev. Math. Phys., 2014, 26, 1450015, 43 pp.

[13] Duchêne V., Raymond N. Spectral asymptotics of a broken delta interaction. J. Phys. A, 2014, 47, 155203, $19 \mathrm{pp}$.

[14] Exner P. An isoperimetric problem for leaky loops and related mean-chord inequalities. J. Math. Phys., 2005, 46, 062105, $10 \mathrm{pp}$.

[15] Exner P., Fraas M. On geometric perturbations of critical Schrödinger operators with a surface interaction. $J$. Math. Phys., 2009, 50, 112101, 12 pp.

[16] Exner P., Ichinose T. Geometrically induced spectrum in curved leaky wires. J. Phys. A, 2001, 34, P. 14391450.

[17] Exner P., Kondej S. Bound states due to a strong $\delta$ interaction supported by a curved surface. J. Phys. A, 2003, 36, P. 443-457.

[18] Exner P., Kondej S. Scattering by local deformations of a straight leaky wire. J. Phys. A, 2005, 38, P. 48654874.

[19] Exner P., Pankrashkin K. Strong coupling asymptotics for a singular Schrödinger operator with an interaction supported by an open arc. Comm. Partial Differential Equations, 2014, 39, P. 193-212.

[20] Exner P., Yoshitomi K. Asymptotics of eigenvalues of the Schrödinger operator with a strong $\delta$-interaction on a loop. J. Geom. Phys., 2002, 41, P. 344-358.

[21] Kondej S., Lotoreichik V. Weakly coupled bound state of 2-D Schrödinger operator with potential-measure. J. Math. Anal. Appl., 2014, 420, P. 1416-1438.

[22] Kondej S., Veselič I. Lower bounds on the lowest spectral gap of singular potential Hamiltonians. Ann. Henri Poincaré, 2007, 8, P. 109-134.

[23] Mantile A., Posilicano A., Sini M. Self-adjoint elliptic operators with boundary conditions on not closed hypersurfaces. ArXiv:1505.07236, 2015.

[24] Popov I. Yu. The operator extension theory, semitransparent surface and short range potential. Math. Proc. Camb. Phil. Soc., 1995, 118, P. 555-563. 
[25] Stollmann P., Voigt J. Perturbation of Dirichlet forms by measures. Potential Anal., 1996, 5, P. $109-138$.

[26] Brown B. M., Grubb G., Wood I. G. $M$-functions for closed extensions of adjoint pairs of operators with applications to elliptic boundary problems. Math. Nachr., 2009, 282, P. 314-347.

[27] Grubb G. A characterization of the non-local boundary value problems associated with an elliptic operator. Ann. Scuola Norm. Sup. Pisa (3), 1968, 22, P. 425-513.

[28] Malamud M. M. Spectral theory of elliptic operators in exterior domains. Russ. J. Math. Phys., 2010, 17, P. $96-125$.

[29] Behrndt J., Langer M. Boundary value problems for elliptic partial differential operators on bounded domains. J. Funct. Anal., 2007, 243, P. 536-565.

[30] Behrndt J., Langer M. Elliptic operators, Dirichlet-to-Neumann maps and quasi boundary triples, in: Operator Methods for Boundary Value Problems, London Math. Soc. Lecture Note Series, 2012, 404, P. 121-160.

[31] Behrndt J., Langer M., Lotoreichik V. Spectral estimates for resolvent differences of self-adjoint elliptic operators. Integral Equations Operator Theory, 2013, 77, P. 1-37.

[32] Gesztesy F., Mitrea M. Generalized Robin boundary conditions, Robin-to-Dirichlet maps, and Krein-type resolvent formulas for Schrödinger operators on bounded Lipschitz domains. Proc. Sympos. Pure Math., Amer. Math. Soc., Providence, RI, 2008, 79, P. 105-173.

[33] Gesztesy F., Mitrea M. A description of all self-adjoint extensions of the Laplacian and Krein-type resolvent formulas on non-smooth domains. J. Anal. Math., 2011, 113, P. 53-172.

[34] Grubb G. Extension theory for elliptic partial differential operators with pseudodifferential methods. London Math. Soc. Lecture Note Series, 2012, 404, P. 221-258.

[35] Posilicano A. Self-adjoint extensions of restrictions. Oper. Matrices, 2008, 2, P. 1-24.

[36] Posilicano A., Raimondi L. Krein's resolvent formula for self-adjoint extensions of symmetric second-order elliptic differential operators. J. Phys. A, 2009, 42, 015204, 11 pp.

[37] Post O. Boundary pairs associated with quadratic forms. Math. Nachr, 2015, doi: 10.1002/mana.201500048.

[38] Schmüdgen K. Unbounded Self-Adjoint Operators on Hilbert Space, Springer, Dordrecht, 2012.

[39] Behrndt J., Langer M. On the adjoint of a symmetric operator. J. Lond. Math. Soc. (2), 2010, 82, P. 563-580.

[40] Lions J., Magenes E. Non-Homogeneous Boundary Value Problems and Applications I. Springer-Verlag, Berlin-Heidelberg-New York, 1972.

[41] Grubb G. Distributions and Operators. Graduate Texts in Mathematics, vol. 252, Springer, New York, 2009.

[42] Behrndt J., Grubb G., Langer M., Lotoreichik V. Spectral asymptotics for resolvent differences of elliptic operators with $\delta$ and $\delta^{\prime}$-interactions on hypersurfaces. J. Spectral Theory, 2015, 5, P. 697-729. 